\title{
Effects of bundling on the properties of the SPC water model
}

\author{
Marc Fuhrmans · Barbara P. Sanders • \\ Siewert-Jan Marrink · Alex H. de Vries
}

Received: 23 December 2008/Accepted: 13 May 2009/Published online: 31 May 2009

(C) The Author(s) 2009. This article is published with open access at Springerlink.com

\begin{abstract}
Simultaneous representation of molecules at an all-atom and coarse-grained level, as required by multiscaling molecular dynamics simulations, poses problems for the treatment of small molecules. If more than one of these molecules are mapped to a single coarse-grained interaction site, unrestricted movement in the all-atom representation can make a meaningful correspondence of the coordinates between the two representations impossible. Restricting the relative movement of molecules mapped to the same coarse-grained interaction site solves that problem, but will have consequences for the properties of the model. Here we investigate the effects of introducing bundling to the common simple point charge (SPC) water model and present a bundled water model that preserves important properties of SPC water relevant for multiscaling.
\end{abstract}

Keywords Water $\cdot$ Molecular dynamics $\cdot$ Multiscaling

\section{Introduction}

Coarse-grained models have become a powerful tool in molecular dynamics simulations, significantly increasing the accessible system sizes and time scales of simulations [1]. Although the resolution of coarse-grained simulations

Dedicated to Professor Sandor Suhai on the occasion of his 65th birthday and published as part of the Suhai Festschrift Issue.

M. Fuhrmans - B. P. Sanders - S.-J. Marrink ·

A. H. de Vries $(\square)$

Groningen Biomolecular Sciences and Biotechnology Institute and Zernike Institute for Advanced Materials,

University of Groningen, Nijenborgh 4,

9747 AG Groningen, The Netherlands

e-mail: A.H.de.Vries@rug.nl is sufficient for a broad range of applications, for some questions the resolution of an all-atom model is required. However, the higher resolution of the fine-grained representation is often not needed for the entire system, or not necessary for the entire duration of the simulation. For these cases, multiscaling methods are being developed in which different parts of the system are represented in different detail or the level of detail can be smoothly switched from coarse-grained to all-atom and vice versa [2].

The current approaches to accomplish this involve treating the system at both levels of detail simultaneously and require a mapping of atoms of the fine-grained representation to the interaction sites of the coarse-grained representation, where the coordinates of those coarsegrained beads are set to be identical to the center-of-mass of the corresponding fine-grained atoms (for examples see publications on spatial multiscaling, e.g. [3], and Hamiltonian exchange, e.g. [4]). Naturally, this leads to problems when several small molecules are mapped to the same coarse-grained bead because the correspondence of the coordinates between the representations becomes meaningless when the fine-grained molecules diffuse too far away from each other. It therefore becomes necessary to restrict the relative movement of small molecules that are mapped to the same coarse-grained interaction site for the duration of the simultaneous simulation.

Solvent is the most commonly needed small molecule in molecular dynamics simulations, making water an ideal candidate for studying the effects that introduction of bundling has on the properties of the model. Here we present a water model based on the widely used simple point charge (SPC) water model [5], restricting the relative movement within groups of four water molecules as required for multiscaling simulations based on the MARTINI coarse-grained model [6]. A number of properties of 
the bundled model important for multiscaling are compared to the properties of SPC water and the MARTINI model. The bundled water model should, however, also be applicable in combination with other coarse-grained models based on a 4-to-1 mapping.

\section{Methods}

\subsection{General simulation setup}

All simulations were performed using the GROMACS software package version 3.3.1 [7] employing the leapfrog integrator.

The simulations are either purely (bundled) fine-grained or coarse-grained. No multiscaling simulations or translations from coarse- to fine-grained or vice-versa are reported in this publication.

\subsubsection{All-atom simulations}

The fine-grained simulations are based on the GROMOS $53 \mathrm{a} 6$ force field [8] using a timestep of $2.5 \mathrm{fs}$. Non-bonded interactions were calculated using a twin-range cut-off scheme. Lennard-Jones and Coulomb interactions within $0.9 \mathrm{~nm}$ were evaluated every time-step based on a neighbor list updated every 5 steps. Interactions beyond that cut-off but within $1.4 \mathrm{~nm}$ were calculated at every update of the neighbor list and assumed constant until the next update. For electrostatic interactions beyond $1.4 \mathrm{~nm}$ a reaction field correction [9] was included with a dielectric constant of 54.

Except noted otherwise, the simulations were carried out in a cubic simulation box under periodic boundary conditions at isotropic pressure coupling using the Berendsen scheme [10] with a reference pressure of 1 bar, a coupling time constant of $0.5 \mathrm{ps}$ and a compressibility of $4.6 \times 10^{-4}$ $\mathrm{bar}^{-1}$. The temperature was coupled to 298 or $323 \mathrm{~K}$ using the Berendsen thermostat [10] with a coupling time constant of 0.1 ps. Simulations were performed starting from an equilibrated system of 1,068 water molecules.

In the simulations using the unmodified SPC water model the water geometry was constrained using the SETTLE algorithm [11]. This was no longer possible in the bundled models and the equivalent rigid-body geometry of water was achieved by using the LINCS algorithm [12] to constrain the atoms of the individual water molecules to a distance of $0.1 \mathrm{~nm}$ between oxygen and hydrogen and $0.1633 \mathrm{~nm}$ between the two hydrogen atoms.

Two applications using the bundled water models were performed. A lipid bilayer consisting of 36 dipalmitoylphosphatidylcholine (DPPC) molecules solvated with 40 water molecules (10 bundles of 4 water molecules) per lipid was simulated at a temperature of $323 \mathrm{~K}$ under semiisotropic pressure coupling conditions. The model for DPPC is a development version of the GROMOS 53a6 force field for lipids [13]. It has been shown that a system of this size is sufficient to yield representative bilayer properties [14]. The starting configuration was taken from an equilibrated system using SPC water. Water was bundled by applying the restraints as appropriate for the bundled models in stages. Short simulations of 10-100 ps were performed during which the bundles formed from the dispersed water molecules without noticeable artifacts. Once all water bundles were formed, production runs of $25 \mathrm{~ns}$ were started.

The second application is that of a protein in water. Hen Egg-White Lysozyme (Protein Data Bank entry 1AKI [15]) was solvated in water. Bundling the water was performed in stages with the protein atoms frozen in place to avoid deformation. Energy minimization and a short equilibration run $(10 \mathrm{ps})$ set the system up for production. Production runs were analyzed over the first $10 \mathrm{~ns}$ of simulation.

Additional changes made to the SPC water model in the process of bundling are documented in Sect. 3 .

\subsubsection{Coarse-grained simulations}

The coarse-grained simulations were made using the MARTINI model [6] using a timestep of $40 \mathrm{fs}$. LennardJones and Coulomb interactions were calculated every step for atoms within $1.2 \mathrm{~nm}$ according to a neighbor list updated every 10 steps. Both the Lennard-Jones and Coulomb potential were modified with a shift function to smoothly reduce them to zero at the cut-off. Electrostatic interactions were screened with an effective dielectric constant of $15 .^{1}$

Except noted otherwise, the simulations were carried out in a cubic simulation box under periodic boundary conditions at isotropic pressure coupling using the Berendsen scheme [10] with a reference pressure of 1 bar, a coupling time constant of $1.2 \mathrm{ps}$ and a compressibility of $5.0 \times 10^{-5} \mathrm{bar}^{-1}$. The temperature was coupled to 298 or $323 \mathrm{~K}$ using a Berendsen thermostat [10] with a coupling time constant of 0.5 ps. Simulations were performed starting from an equilibrated system of 267 water beads (corresponding to 1,068 water molecules).

\subsection{Determination of specific properties}

\subsubsection{Diffusion coefficients}

Data was gathered over $5 \mathrm{~ns}$ for the fine-grained systems and over $40 \mathrm{~ns}$ for the coarse-grained comparison with an output-frequency of 1 per $2.5 \mathrm{ps}$ for both.

\footnotetext{
${ }^{1}$ Details of the MARTINI model can be found in the original publication [6].
} 
The center-of-mass coordinates were extracted from the trajectory for all objects (molecules or bundles or coarsegrained beads) in the system and used to calculate the mean-square displacement (MSD, $\left\langle r^{2}\right\rangle$ ). A final plot of the average MSD and of the standard-error in the MSD as a function of time $t$ was obtained by collecting the statistics over all molecules. Using the Einstein relation

$\left\langle r^{2}\right\rangle=6 D t+C$,

the diffusion coefficient $D$ was obtained as one sixth of the slope of the plot. This was done by fitting a linear function to the MSD plot starting from $t=50 \mathrm{ps}$ up to the maximum time at which both the plot of the mean-square displacement and the standard-error still appear linear (0.3 ns). Error values were obtained in the same manner as one sixth of the slope of the standard-error plot.

\subsubsection{Viscosity}

The shear viscosity was calculated using the Einstein method relating the shear viscosity to the time autocorrelation of the off-diagonal elements of the stress tensor $\sigma$ [16].

$\eta=\frac{V}{2 k_{B} T} \frac{\mathrm{d}}{\mathrm{d} t}\left\langle\left[\int_{0}^{t} \sigma\left(t^{\prime}\right) \mathrm{d} t^{\prime}\right]^{2}\right\rangle(t \rightarrow \infty)$

The data was obtained from 2.5 ns of simulation, writing the stress data every $10 \mathrm{fs}$.

\subsubsection{Rotational autocorrelation}

500 ps of simulation with an output-frequency of 1 per 5 fs were analyzed.

Specifically, the orientation of the vector defined by the cross-product of the two vectors corresponding to the $\mathrm{O}-\mathrm{H}$ bonds was correlated using the cosine of the angle between the orientations at different time points. The autocorrelation was obtained for each molecule individually and averaged over groups of 12 water molecules before fitting to the bi-exponential decay-function:

$f(t)=c \mathrm{e}^{-t / \tau_{1}}+(1-c) \mathrm{e}^{-t / \tau_{2}}$.

Final values of the fitted parameters were obtained as the average over all groups.

\subsubsection{Surface tension}

The simulations were started from an equilibrated system of 2,136 water molecules in a rectangular simulation box of $3.2292 \times 3.2292 \times 12.9 \mathrm{~nm}^{3}$ with the water molecules forming a continuous layer in the $x y$-plane but well separated from their periodic images in the $z$-direction. The simulations were run at constant volume for $1 \mathrm{~ns}$ generating output every $10 \mathrm{fs}$.

The surface tension $\gamma$ was then calculated according to

$\gamma=L_{z}\left(P_{z}-\frac{P_{x}+P_{y}}{2}\right) \frac{1}{2}$,

where $L_{z}$ is the box-length in the $z$-direction and $P_{x}, P_{y}$ and $P_{z}$ are the time-averaged diagonal elements of the pressure tensor in the $x, y$ and $z$ dimension, respectively. The factor $1 / 2$ stems from the fact that there are two surfaces in the system.

\subsubsection{Free energies of hydration}

Hydration free energies $\Delta G_{\text {hyd }}$ were obtained for butane, ethanol and the $\mathrm{Na}^{+}$and $\mathrm{Cl}^{-}$ions using thermodynamic integration [17]. Several $1 \mathrm{~ns}$ simulations were performed in which the interactions of the solute with the solvent were scaled with a constant factor $\lambda$ with values from 0 (full interaction) to 1 (no interactions) recording the $\mathrm{d} H / \mathrm{d} \lambda$ values every 2.5 fs (where $H$ is the enthalpy). Simulations were performed for $\lambda$ values spaced 0.1 apart. Depending on the different solutes, additional simulations were performed in regions where $\mathrm{d} H / \mathrm{d} \lambda$ varies strongly as a function of $\lambda$.

For each $\lambda$ value the average $\mathrm{d} H / \mathrm{d} \lambda$ value and error estimate were obtained, both of which were plotted against $\lambda$. The free energy differences $\Delta G$ between interacting and non-interacting solute and the corresponding errors were obtained as the integral of the respective plots.

For each solute the process was performed in vacuum $\left(\Delta G_{\mathrm{vac}}\right)$ and in each of the water models $\left(\Delta G_{\mathrm{sol}}\right)$. The free energy of hydration in each water model was then obtained as

$\Delta G_{\mathrm{hyd}}=\Delta G_{\mathrm{vac}}-\Delta G_{\mathrm{sol}}$,

and the error value as the sum of the errors in $\Delta G_{\mathrm{vac}}$ and $\Delta G_{\mathrm{sol}}$.

\subsubsection{Error values}

For average properties calculated from a time series the given error values are error estimates obtained via blockaveraging [18]. For properties averaged over an ensemble of $N$ molecules or groups of molecules, the standard-error is given.

\section{Parametrization of the bundled SPC water model}

\subsection{The bundling potential}

The effect that has to be accomplished by the bundling is to keep the clustered molecules as first neighbors. Otherwise, mixing water bundles could have very close (or identical) 
centers-of-mass, giving rise to overlapping interaction sites in the coarse-grained representation.

The bundling is achieved via the introduction of attractive harmonic potentials between all pairs in the groups of clustered water molecules, causing the clusters to adopt a roughly tetrahedral shape. Since the equilibrium distance for first neighbors in the SPC water model is approximately $0.28 \mathrm{~nm}$, it makes little sense to have the potentials already affect the molecules within that distance and we chose to have the potentials start at an onset of $0.3 \mathrm{~nm}$. At that distance, the potential starts at zero and continues according to

$V_{\text {bundling }}=\frac{1}{2} k_{\mathrm{dr}}\left(r_{i j}-0.3 \mathrm{~nm}\right)^{2}$

in which $k_{\mathrm{dr}}$ is the force-constant and $r_{i j}$ is the distance between the oxygen atoms.

\subsection{Reproducing the density}

The most important property to reproduce is the density. We therefore chose to model the bundled water to have a density of $952.3 \mathrm{~kg} \mathrm{~m}^{-3}$ which is the density of SPC water at $323 \mathrm{~K}^{2}$ under the conditions of our simulations. The introduction of attractive potentials will increase the density of the model, with the extent of the increase depending on the strength of the force-constant.

Figure 1 shows the effect of the restraining force-constant $k_{\mathrm{dr}}$ on the density for SPC water at $323 \mathrm{~K}$. The increase of the density is significant, with a high forceconstant effecting a greater increase than a small one. However, lowering the force-constant will only reproduce the original density at zero. Therefore, a compensating interaction to decrease the density is needed. This was initially attempted by increasing the distance of water molecules within one cluster without directly affecting the interactions of molecules belonging to different clusters. Unfortunately, introducing a repulsive harmonic potential between the oxygen atoms or alternatively increasing the $\mathrm{C}_{12}$-parameter of the Lennard-Jones potential

$V_{\mathrm{LJ}}\left(r_{i j}\right)=\frac{C_{12}}{r_{i j}^{12}}-\frac{C_{6}}{r_{i j}^{6}}$

from its original value of $2.634129 \times 10^{-6} \mathrm{~kJ} \mathrm{~mol}^{-1}$ $\mathrm{nm}^{+12}$ for the oxygen-oxygen interaction within the bundled clusters caused the clustered molecules to become second neighbors, i.e. separated from the rest of the bundled molecules by molecules from another bundle, before the desired density was reached (data not shown). We

\footnotetext{
$2323 \mathrm{~K}$ was chosen because it is a standard temperature for many lipid systems which are of special interest to the authors.
}

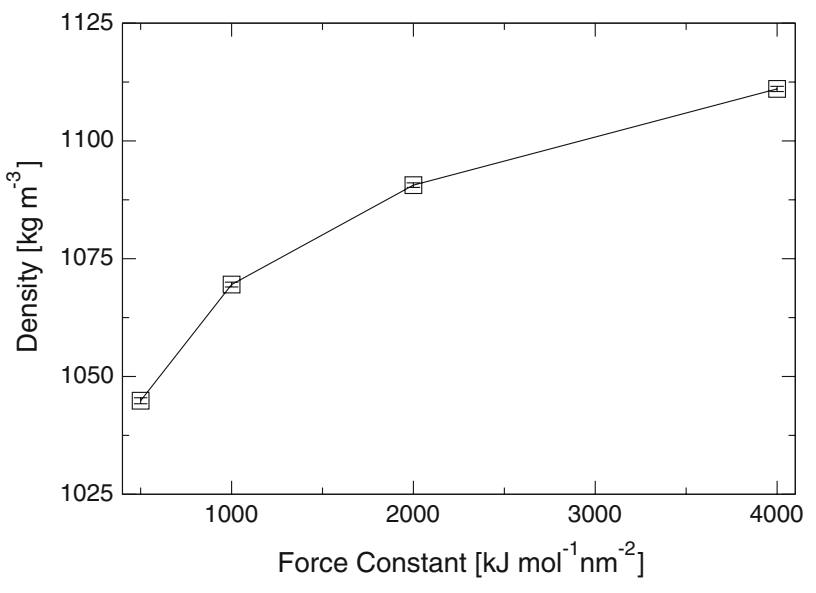

Fig. 1 The effects of bundling on the density of water at $323 \mathrm{~K}$ as a function of the strength of the introduced restraining potential. The density of SPC water under the simulated conditions is $952.3 \mathrm{~kg} \mathrm{~m}^{-3}$. The line between the calculated points serves to guide the eye

therefore decided to increase the $\mathrm{C}_{12}$-parameter of the Lennard-Jones interaction between all water oxygen atoms.

In Fig. 2 the dependence of the density on the restraining force-constant is shown for a number of $\mathrm{C}_{12^{-}}$ parameters. According to these results, a force-constant of $500 \mathrm{~kJ} \mathrm{~mol}^{-1} \mathrm{~nm}^{-2}$ (or even lower) would appear to be a good choice for a bundled water model, but closer examination revealed that under these conditions clustered water molecules occasionally become second neighbors. We therefore focused on the force-constants of 1,000 and $4,000 \mathrm{~kJ} \mathrm{~mol}^{-1} \mathrm{~nm}^{-2}$ and performed additional simulations varying the $\mathrm{C}_{12}$-parameters around the values that had the density closest to that of SPC water. A plot of the observed densities is shown in Fig. 3.

The two models with the best matching densities were chosen to further study the effects of bundling and shall be referred to as models 1 and 2, respectively (see Table 1 for the exact parameters used). Additional simulations at $298 \mathrm{~K}$ show that the agreement between the densities of the models is also preserved at that temperature (data not shown).

\section{Effects of the bundling}

\subsection{Radial distribution}

The radial distribution $g(r)$ provides valuable information on the structure of water in the different models. Figure 4 shows the radial distribution for oxygen atoms of all water molecules in the system (top) and for only the oxygens within one cluster of bundled water molecules (bottom) at $323 \mathrm{~K}$. In the bundled models, the distribution is shifted towards larger radii, as would be expected from the 


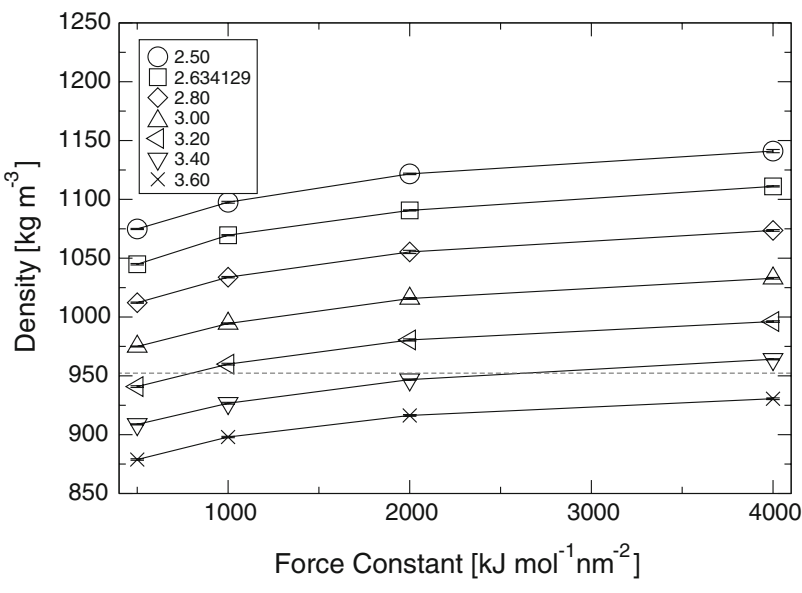

Fig. 2 Density of water as a function of the strength of the bundling potential for different Lennard-Jones $\mathrm{C}_{12}$-parameters given in the legend in units of $10^{-6} \mathrm{~kJ} \mathrm{~mol}^{-1} \mathrm{~nm}^{+12}$. The horizontal dashed line indicates the density of SPC water under the simulated conditions. Lines between calculated points are meant to guide the eye

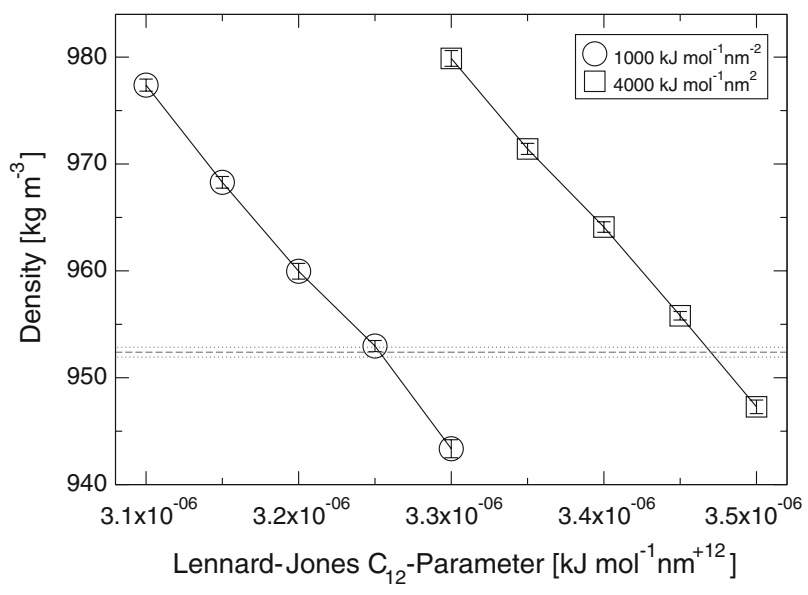

Fig. 3 Finetuning of the Lennard-Jones $C_{12}$-parameters for the models with a force-constant of $1,000 \mathrm{~kJ} \mathrm{~mol}^{-1} \mathrm{~nm}^{-2}$ (circles) and $4,000 \mathrm{~kJ} \mathrm{~mol}^{-1} \mathrm{~nm}^{-2}$ (squares), respectively. The horizontal dashed lines indicate the $67 \%$ confidence interval of the density of SPC water under the simulated conditions. Lines between calculated points serve as guides to the eye

Table 1 Overview of the parameters changed with respect to SPC water in the models used to study the effects of bundling $\left(k_{\mathrm{dr}}\right.$ : Force constant of the introduced restraining force, $C_{12}: C_{12}$-parameter of the Lennard-Jones interaction between oxygen atoms)

\begin{tabular}{lll}
\hline & $k_{\mathrm{dr}}\left(\mathrm{kJ} \mathrm{mol}^{-1} \mathrm{~nm}^{-2}\right)$ & $C_{12}\left(\mathrm{~kJ} \mathrm{~mol}^{-1} \mathrm{~nm}^{+12}\right)$ \\
\hline Model 1 & 1,000 & $3.25 \times 10^{-6}$ \\
Model 2 & 4,000 & $3.45 \times 10^{-6}$ \\
SPC & $\mathrm{n} / \mathrm{a}$ & $2.634129 \times 10^{-6}$ \\
\hline
\end{tabular}

increased Lennard-Jones $\mathrm{C}_{12}$-parameters. While the position of the peak representing the first neighbors is only slightly shifted and still overlaps with the peak from SPC

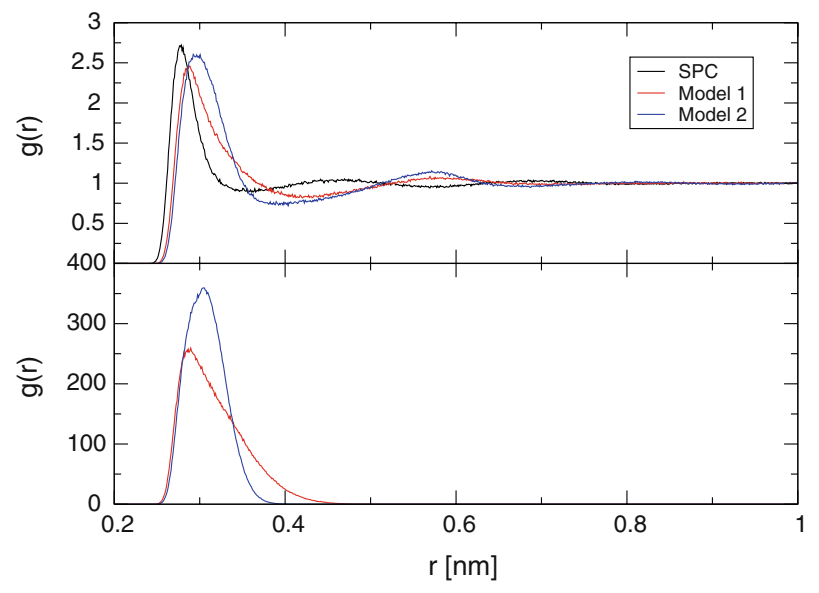

Fig. 4 Radial distribution of oxygen atoms for SPC water (black) and the bundled models 1 (red) and 2 (cyan) at $323 \mathrm{~K}$. The radial distribution is given for all molecules in the system (top) and for the molecules within a single cluster (bottom)

water, the peak corresponding to the second neighbors is shifted much further and lies at approx. $0.58 \mathrm{~nm}$ as opposed to $0.47 \mathrm{~nm}$ in SPC water. In addition, the number of water molecules in the first hydration shell of water is increased in the bundled models compared to SPC.

Comparing the two bundled models, it is found that the first neighbors are on average farther apart in model 2. As seen from the radial distribution for the single clusters, both models keep bundled molecules from becoming second neighbors at all times, with model 2 displaying a more narrow distribution of distances within the bundles, showing the effects of the higher force-constant.

In Fig. 5, the radial distribution of the centers-of-mass of the fine-grained water clusters is compared to the radial distribution of the coarse-grained beads in the MARTINI model at $323 \mathrm{~K}$. Here, the agreement is much better than for the comparison at the level of water molecules. While the first neighbor peak starts at lower radii in the finegrained models due to the relative movement of the bundled water molecules, the maxima appear at similar positions. The peaks for the second neighbors lie at a slightly larger radius for the fine-grained bundles than for the coarse-grained beads, which again can be rationalized as being due to the relative movement still allowed in the bundles. It is interesting to note that similar findings were reported for hexadecane when comparing the MARTINI model to mapped fine-grained hexadecane [19]. One may conclude that the repulsive potential used in the MARTINI model is too steep to faithfully reproduce the effective repulsion between groups of atoms.

Comparing the two fine-grained models, the onset of the first neighbor peak is at a slightly lower distance for model 1 in agreement with the wider distribution of distances at the lower force-constant of this model. 


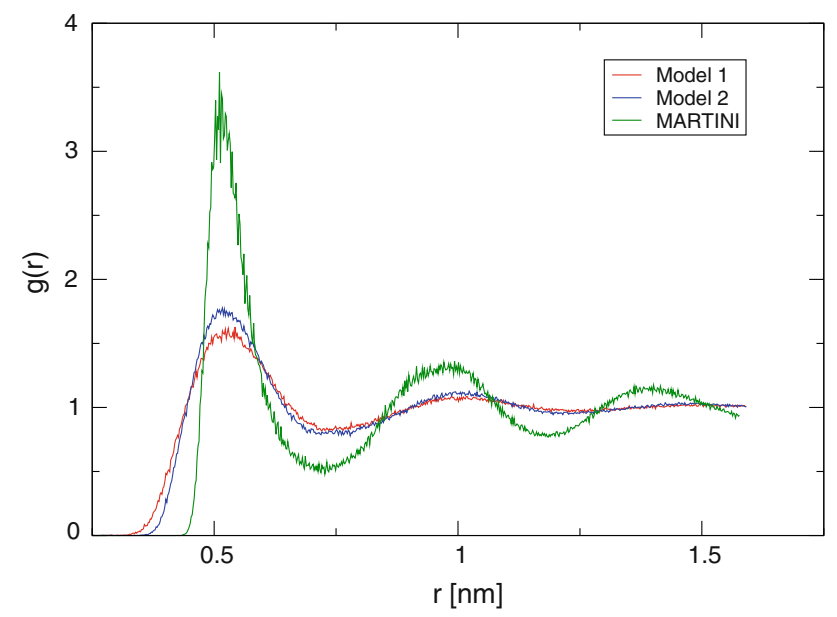

Fig. 5 Comparison of the radial distributions of the centers-of-mass of the bundled water models 1 (red) and 2 (cyan) with the radial distribution of the water beads in the coarse-grained MARTINI model (green) at $323 \mathrm{~K}$

A potential problem for multiscaling simulations is the smallest distance observed between the centers-of-mass for the bundled models. While not visible in Fig. 5, there are sporadic occurrences of distances as low as $0.1 \mathrm{~nm}$ for both models. While these would lead to large forces when directly translated to a coarse-grained representation, it is likely that the forces from the simultaneous coarse-grained representation in an actual multiscaling simulation would prevent them. This is, however, a general problem of multiscaling simulations and not limited to the simulation of small molecules. As such, it will have to be addressed on a different level than the water model.

Simulations at $298 \mathrm{~K}$ show almost identical radial distributions (data not shown).

\subsection{Diffusion}

The diffusion for the water molecules in our model will be influenced strongly by the bundling itself. Since bundled water has to move as a tetramer with a higher hydrodynamic radius and thus larger friction than SPC water, the diffusion coefficients are expected to be lower according to the Stokes-Einstein model. Reproducing the value of SPC water therefore cannot be our intention. However, diffusion can provide information on molecular interactions and is a way to compare the two chosen sample models.

Table 2 shows the observed diffusion coefficients for the different models including the coarse-grained MARTINI model. As predicted, the diffusion coefficients for the water molecules are much lower in the bundled models than in SPC water. However, proper comparison should be made between the diffusion of bundled water and that of four independently diffusing SPC water molecules, which corresponds to the limit of no restraining potential. It can be shown that the diffusion coefficient of SPC water should therefore be divided by four [23], and thus the diffusion coefficients of the bundled water models compare quite well to the reference values of $1.05 \times 10^{-9} \mathrm{~m}^{2} \mathrm{~s}^{-1}$ at $298 \mathrm{~K}$ and $1.55 \times 10^{-9} \mathrm{~m}^{2} \mathrm{~s}^{-1}$ at $323 \mathrm{~K}$. The fact that the bundled molecules diffuse slightly faster than independent molecules is likely caused by the coordinated movement enforced by the bundling.

Comparing the two bundled models, the values for the diffusion coefficients lie within each other's confidence intervals. Looking at the trend of the diffusion coefficients $D$ with temperature our results are found to be in qualitative agreement with the Stokes-Einstein relation

$D=\frac{k_{B} T}{6 \pi \eta r}$.

It anti-correlates well with the viscosity $\eta$, which is also reported for the different models in Table 2. The hydrodynamic radius $r$, as reflected by the similar radial distributions, does not change much with the temperature and therefore does not explain the observed changes in diffusion.

\subsection{Rotational autocorrelation}

After studying the translation of water molecules in the different models, rotational movement was examined, which gives us further insight into the interactions between the molecules in the bundled water models. Generally, two

Table 2 Diffusion coefficients $D$, viscosities $\eta$, and surface tensions $\gamma$ of the different water models at 298 and $323 \mathrm{~K}$

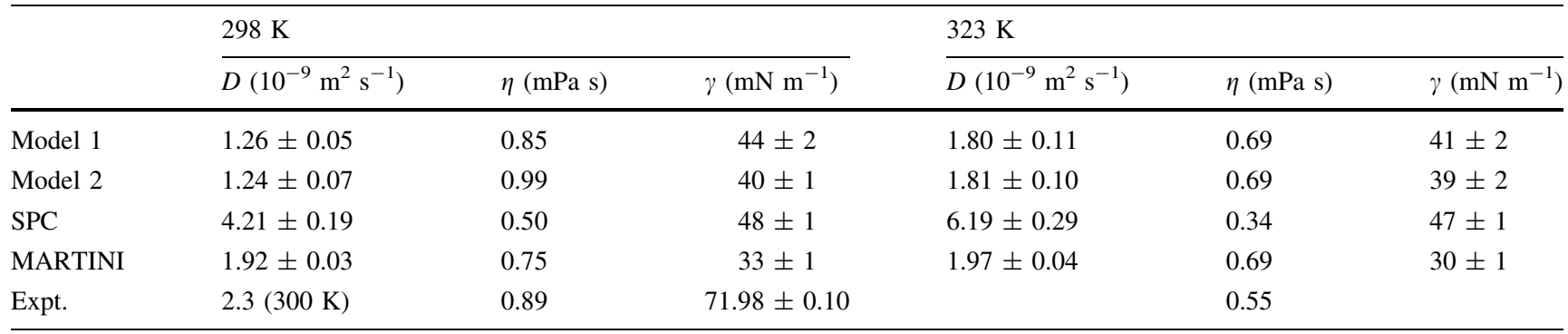

For comparison experimental values at $298 \mathrm{~K}$ are given for $D$ [20], $\eta$ [21], and $\gamma$ [22] 
kinds of rotation can be distinguished for molecules in solution-the rapid rotational vibrations between collisions and the slower tumbling, with which the orientation of molecules changes over longer times as the net-result of the faster rotations. We therefore chose the bi-exponential decay-function Eq. 3 as a model for the autocorrelation of the orientation. The parameter $\tau_{1}$ dominates the behavior for short times $t$ and represents the rapid rotations, whereas the parameter $\tau_{2}$ becomes more important at longer times and can be interpreted as the decay of orientational correlation via tumbling.

A graph of the rotational autocorrelation is given in Fig. 6 and Table 3 shows the fitting parameters that best match the data obtained for our models and SPC water. The values of the parameters $\tau_{1}$ and $c$ are very close for the different models. Since the tumbling movements and in correspondence $\tau_{2}$ are more sensitive to the inter-molecular interactions we will focus our discussion on this parameter. Note that our value for SPC water $\left(\tau_{2}=2 \mathrm{ps}\right)$ is slightly higher than that found by van der Spoel et al. (1.6 ps) [24]. They used a slightly smaller long-range cut-off value of $1.2 \mathrm{~nm}$. Comparison to experiment is complicated because different relaxation mechanisms may contribute to a particular measurement. Our value is quite close to the value of 2.4 ps determined by Halle and Wennerström obtained from measuring quadrupolar relaxation using NMR [25].

Rotation is faster in the bundled models indicating a lower friction with that effect being more pronounced in model 2. Looking at the radial distribution (Fig. 4, top), it is clear that the water molecules are on average farther apart in the bundled models than in the SPC model reflecting the larger Van-der-Waals radius due to the increased $\mathrm{C}_{12}$-parameter. Taking into account that there are no Van-der-Waals interactions for hydrogen atoms, rotation is almost exclusively affected by electrostatic interactions and a higher distance between the water molecules

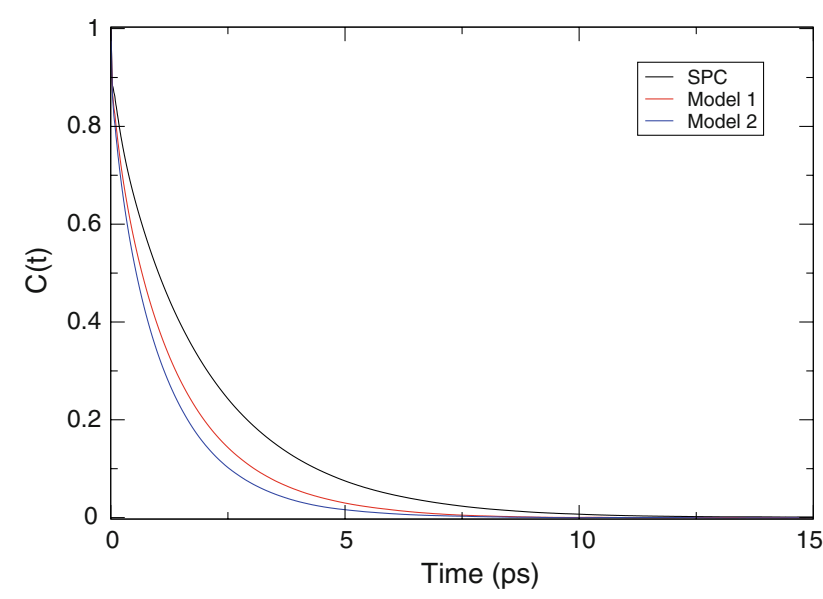

Fig. 6 Average rotational autocorrelation for the different models at $298 \mathrm{~K}$ thus entails weaker electrostatic interactions and thereby faster rotations. In addition, dipole-dipole electrostatic interactions decay more rapidly than the number of interactions increases with distance, making the position of the first peak in the radial distribution the most significant for the assessment of the strength of these interactions. Judging from the radial distribution one would thus expect rotations to be faster in the bundled models with model 2 being faster than model 1 , which rationalizes our results.

\subsection{Surface tension}

When a simulated system includes an interface separating an aqueous region from another, the energy costs associated with maintaining that interface become important. In our simulations we measured the surface tension of a water-vacuum interface.

Table 2 shows the surface tension $\gamma$ obtained for our models and SPC water. Both bundled models have a lower surface tension than SPC water with model 2 being slightly lower than model 1. Since, like rotational friction, the surface tension of water is mostly determined by electrostatic interactions, this finding is consistent with our interpretation of weaker electrostatic interactions due to an increase of the average distance of the first neighbors.

While the reduced surface tension might affect the outcome of simulations, it is of less importance for the purpose of multiscaling simulations based on the MARTINI model, in which the surface tension is even lower than in the bundled models.

\subsection{Hydration free energy}

The interactions of water with other molecules is also an important property to reproduce. Free energies of solvation are a good indicator for changes in these interactions in the bundled water models and directly influence the behavior of solutes. Table 4 shows the hydration free energies for ethanol and butane as examples of organic molecules of different hydrophobicity, and for the $\mathrm{Na}^{+}$and $\mathrm{Cl}^{-}$ions.

The results show very good agreement between the hydration free energies of ethanol and butane in bundled and in SPC water. Since the interactions of the water molecules with the solute have not been changed directly, the observed changes depend solely on the changed interactions of water with itself, showing that the changes in structure as seen in the radial distributions do not affect the interaction with solutes too strongly. For the charged species, the effects of bundling on the hydration free energy are seen to be considerably larger than for the neutral species. The ordering effect of the ions on water is strong and the perturbed water-water interactions are likely to affect the enthalpies and entropies associated with ion 
Table 3 Rotational autocorrelation-fitting parameters for the bi-exponential decay-function Eq. 3 of the different water models at 298 and $323 \mathrm{~K}$

\begin{tabular}{|c|c|c|c|c|c|c|}
\hline & \multicolumn{3}{|l|}{$298 \mathrm{~K}$} & \multicolumn{3}{|l|}{$323 \mathrm{~K}$} \\
\hline & $c$ & $\tau_{1}$ & $\tau_{2}$ & $c$ & $\tau_{1}$ & $\tau_{2}$ \\
\hline Model 1 & $0.22 \pm 0.02$ & $0.11 \pm 0.02$ & $1.40 \pm 0.02$ & $0.21 \pm 0.01$ & $0.07 \pm 0.01$ & $1.04 \pm 0.01$ \\
\hline Model 2 & $0.20 \pm 0.01$ & $0.07 \pm 0.01$ & $1.19 \pm 0.01$ & $0.22 \pm 0.01$ & $0.08 \pm 0.01$ & $0.85 \pm 0.01$ \\
\hline SPC & $0.22 \pm 0.04$ & $0.20 \pm 0.04$ & $1.97 \pm 0.03$ & $0.21 \pm 0.02$ & $0.10 \pm 0.02$ & $1.34 \pm 0.02$ \\
\hline
\end{tabular}

Table 4 Free energies of hydration $\Delta G_{\text {hyd }}$ of butane, ethanol and the $\mathrm{Na}^{+}$and $\mathrm{Cl}^{-}$ ions for the different water models at $298 \mathrm{~K}$ (top) and $323 \mathrm{~K}$ (bottom)

For comparison the values for the hydration of the corresponding coarse-grained beads in MARTINI water and experimental values for butane and ethanol [26] are also given

\begin{tabular}{|c|c|c|c|c|}
\hline & \multicolumn{4}{|c|}{$\Delta G_{\mathrm{hyd}}\left[\mathrm{kJ} \mathrm{mol}^{-1}\right]$} \\
\hline & Butane & Ethanol & $\mathrm{Na}^{+}$ & $\mathrm{Cl}^{-}$ \\
\hline Model 1 & $+6.6 \pm 1.3$ & $-23.0 \pm 1.4$ & $-395.7 \pm 1.1$ & $-380.6 \pm 1.9$ \\
\hline Model 2 & $+5.7 \pm 1.4$ & $-24.4 \pm 1.2$ & $-397.3 \pm 0.2$ & $-382.8 \pm 1.9$ \\
\hline SPC & $+6.6 \pm 1.4$ & $-23.2 \pm 1.0$ & $-389.0 \pm 1.1$ & $-370.6 \pm 1.5$ \\
\hline MARTINI & $+8.7 \pm 0.4$ & $-15.5 \pm 0.5$ & & \\
\hline Expt. & +8.7 & -20.9 & & \\
\hline Model 1 & $+9.0 \pm 1.2$ & $-21.4 \pm 1.1$ & $-392.6 \pm 1.0$ & $-371.6 \pm 1.2$ \\
\hline Model 2 & $+7.2 \pm 1.0$ & $-22.1 \pm 1.1$ & $-394.2 \pm 1.1$ & $-372.7 \pm 1.3$ \\
\hline SPC & $+8.0 \pm 1.2$ & $-20.8 \pm 0.9$ & $-386.0 \pm 1.0$ & $-368.2 \pm 1.2$ \\
\hline MARTINI & $+11.0 \pm 0.4$ & $-11.0 \pm 0.5$ & & \\
\hline
\end{tabular}

hydration. Comparing radial distributions of the water $\mathrm{O}$ - and $\mathrm{H}$-atoms with respect to the ions between the models does, however, not present a clear clue to the observed increased hydration of the ions in the bundled water models as compared to SPC water (data not shown).

\subsection{Applications}

To demonstrate the usefulness of the models in biomolecular systems two types of systems were investigated. The first application is that of a lipid bilayer in water. Figure 7 shows the electron density profile across a bilayer for a DPPC bilayer in water. The total profile as well as the contributions of the water and lipids are shown. The profile gives insight into a number of important properties characterizing lipid bilayers and the total electron density profile can be obtained from electron scattering data [27]. The thickness of the bilayer is usually defined as the distance between the peaks in the electron density profile. It is seen from Fig. 7 that the thickness of the bilayer decreases somewhat when using the bundled water. The decrease in thickness is accompanied by an increase in area per lipid (SPC: $0.62 \pm 0.07 \mathrm{~nm}^{2}$, model 1: $0.67 \pm 0.05 \mathrm{~nm}^{2}$, model 2: $0.72 \pm 0.02 \mathrm{~nm}^{2}$ ) and therefore an increase in the average distance between lipid head groups. Also, the water is seen to penetrate more deeply into the lipid bilayer interface. These effects may be rationalized in terms of the interactions between the bundles of water which are more

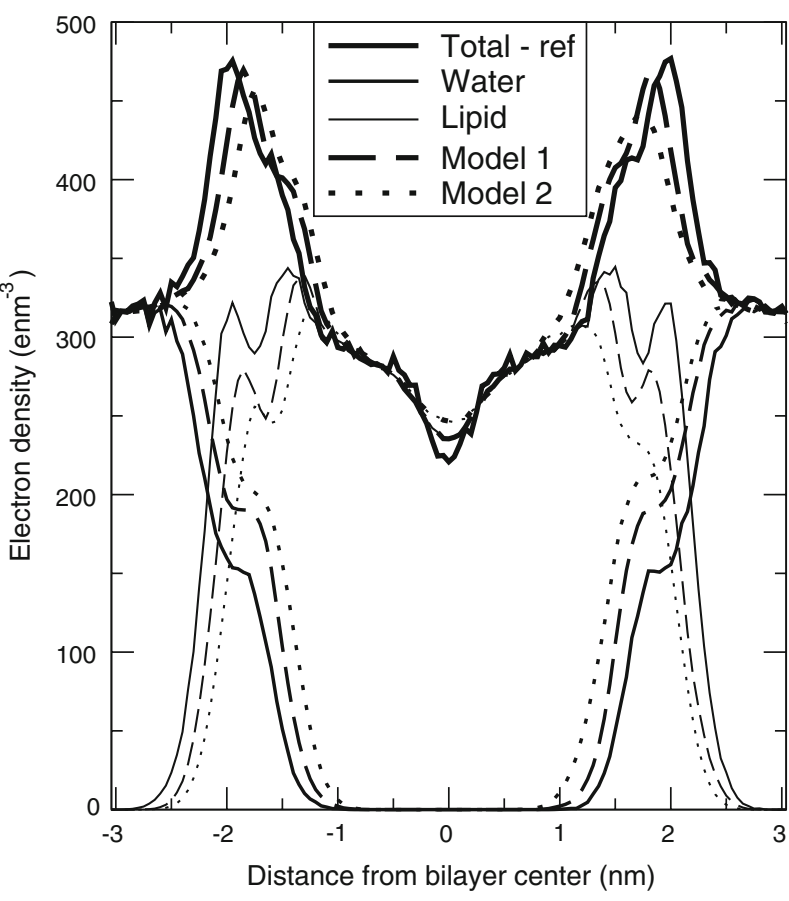

Fig. 7 The effects of bundling on the electron density profile across a DPPC bilayer in water at $323 \mathrm{~K}$. The total profiles (thickest lines) are shown, as well as the contributions of water (intermediate lines) and lipids (thinnest lines). The profiles for normal SPC water are shown in drawn lines, long dashed lines are used for model 1 and dotted lines for model 2 


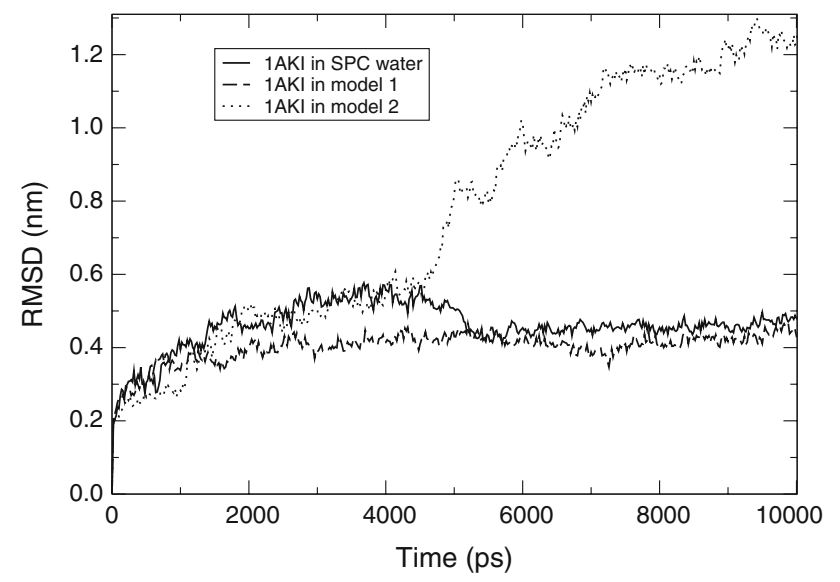

Fig. 8 The effects of bundling on the root mean square distance between the $\mathrm{C} \alpha$ atoms of $1 \mathrm{AKI}$ as a function of time. The RMSD was determined with respect to the same starting structure after obtaining a best fit to the reference structure. SPC water: drawn line; model 1: long dashed line; model 2: dotted line

repulsive than in the case of SPC water. The repulsion between bundles of water may be relieved by interposing lipid head groups. Also, the increased hydration of ionic species (due to the lower hydration free energies) probably contributes to the increased hydration of the head groups.
The second application is that of hen egg-white lysozyme [15] in water. The root mean square deviation (RMSD) of the $\mathrm{C} \alpha$ atoms of the protein structures from the starting structure is shown in Fig. 8. The RMSD evolves similarly in the simulations with SPC water and bundled water model 1 , but dramatically changes after $5 \mathrm{~ns}$ in the simulation using bundled water model 2. Visualization of the protein structure and determination of the secondary structure reveals that the protein behaves similarly in the simulations with SPC water and bundled water model 1, but unfolds in the simulation with bundled water model 2 . The DSSP analysis [28] of the proteins in the different simulations is shown in Fig. 9. The most likely explanation is that the tighter water bundles of model 2 open up the structure of the protein, whereas the less restricted water molecules of model 1 solvate the protein more similarly to SPC water.

\section{Conclusions}

We have shown that it is possible to introduce bundling to the SPC water model by introducing restraining potentials. The resulting increase in density can be compensated by increasing the Lennard-Jones $\mathrm{C}_{12}$-parameter.
Fig. 9 The effects of bundling on the secondary structure of $1 \mathrm{AKI}$ as a function of time as calculated using the DSSP analysis. The occurrence of structure elements is indicated by using a color code, which is shown in the figure. a SPC water; b model 1; c model 2
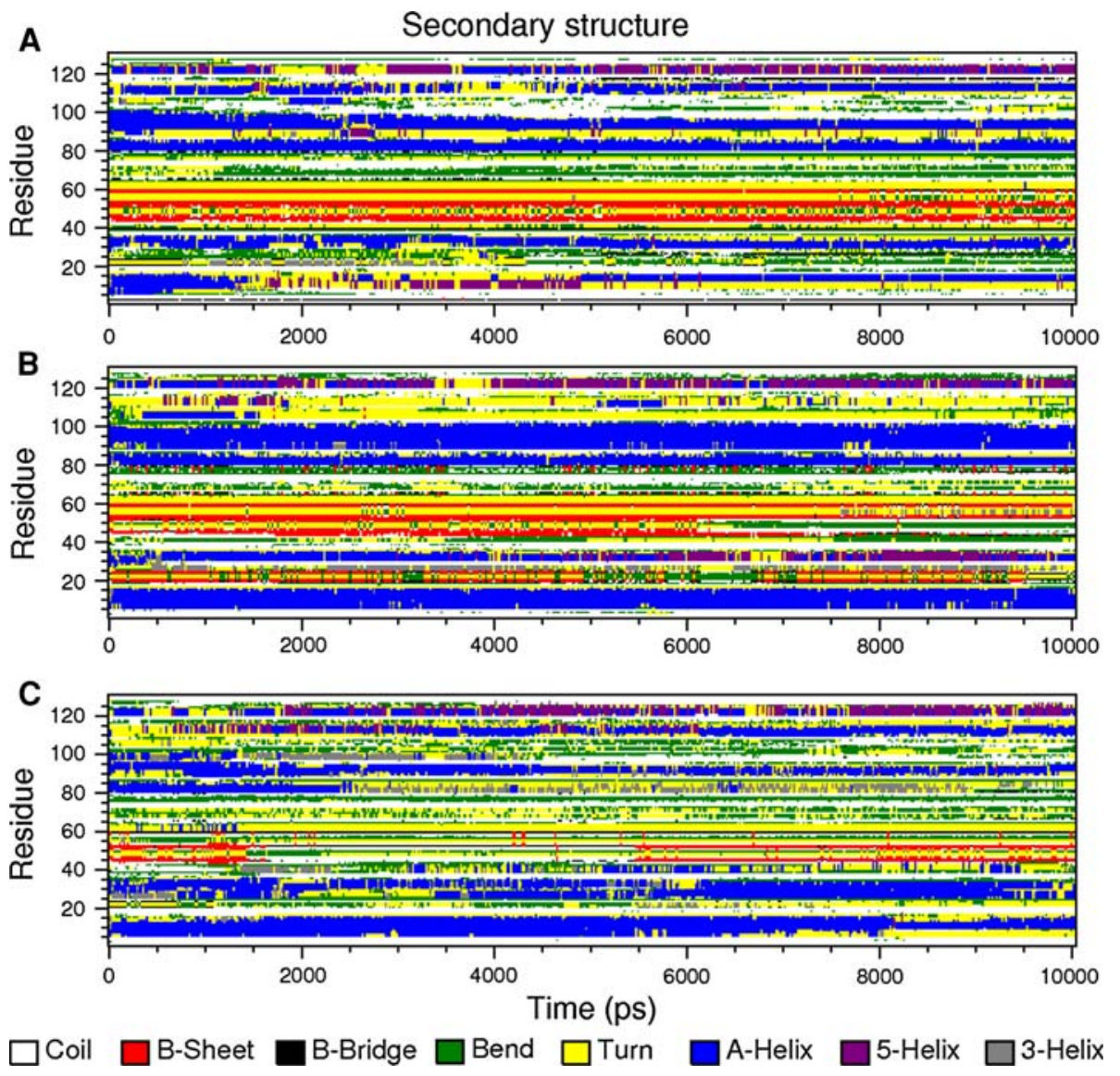
There are many combinations of the force-constant of the bundling potential and the $\mathrm{C}_{12}$-parameter that reproduce the correct density. Model 2 of our tested models keeps the water molecules close to a tetrahedral conformation at all times making overlaps in the coarse-grained representation very unlikely. Model 1 allows more deformations of the water clusters. While preventing clustered molecules from becoming second neighbors at all times, deformations in the models occasionally allow the centers-of-mass of neighboring clusters to become too close for corresponding coarse-grained beads. However, forces from the coarse-grained representation in an actual multiscaling simulation are likely to prevent such conformations in the fine-grained representation in the first place.

Overall, the changes introduced by the bundling most strongly affect the self-interactions of the water molecules, resulting in an altered water structure and as a consequence different dynamics. Interactions with other molecules, however, stay mostly unaltered and with the density and the free energies of hydration of small molecules the most important properties of SPC water are well preserved. Applications to important biomolecular systems show that stronger bundling may lead to some artifacts, exemplified by the larger penetration of water into lipid bilayer interfaces and globular proteins, probably because of increased hydration of ionic species. However, these applications also show that bundled water models are viable in realistic applications and will be important assets in multiscaling simulations.

Our study suggests that a smaller restraining force (and in consequence a smaller required change of the $\mathrm{C}_{12^{-}}$ parameter) will preserve the properties of the SPC better than a large force. The minimal force required to keep the molecules bundled and still prevent overlaps in the corresponding coarse-grained representation of a multiscaling simulation would thus be the best choice for a bundled water model.

Open Access This article is distributed under the terms of the Creative Commons Attribution Noncommercial License which permits any noncommercial use, distribution, and reproduction in any medium, provided the original author(s) and source are credited.

\section{References}

1. Voth GA (2009) Coarse-graining of condensed phase and biomolecular systems. CRC Press, Boca Raton

2. Ayton GS, Noid WG, Voth GA (2007) Curr Opin Struct Biol 17:192-198

3. Ensing B, Nielsen SO, Preston BM, Klein ML, Parrinello M (2007) J Chem Theor Comput 3:1100-1105

4. Christen M, van Gunsteren WF (2006) J Chem Phys 124:154106

5. Berendsen HJC, Postma JPM, van Gunsteren WF, Hermans J (1981) In: Pullman B (ed) Intermolecular forces: proceedngs of the fourteenth Jerusalem symposium on quantum chemistry and biochemistry. Reidel, Dordrecht

6. Marrink SJ, Risselada HJ, Yefimov S, Tieleman DP, de Vries AH (2007) J Phys Chem B 111:7812-7824

7. van der Spoel D, Lindahl E, Hess B, Groenhof G, Mark AE, Berendsen HJC (2005) J Comput Chem 26:1701-1718

8. Oostenbrink C, Villa A, Mark AE, van Gunsteren WF (2004) J Comput Chem 25:1656-1676

9. Tironi IG, Sperb R, Smith PE, van Gunsteren WF (1995) J Chem Phys 102:5451-5459

10. Berendsen HJC, Postma JPM, DiNola A, Haak JR (1984) J Chem Phys 81:3684-3690

11. Miyamoto S, Kollman PA (1992) J Comput Chem 13:952-962

12. Hess B, Bekker H, Berendsen HJC, Fraaije JGEM (1997) J Comp Chem 18:1463-1472

13. de Vries AH (2009) In preparation, topology available on request

14. de Vries AH, Chandrasekhar I, van Gunsteren WF, Hünenberger PH (2005) J Phys Chem B 109:11643-11652

15. Artymiuk PJ, Blake CCF, Rice DW, Wilson KS (1982) Acta Crystallogr Sect B 38:778-783

16. Guo G-J, Zhang Y-G, Zhao Y-J (2003) Phys Rev E 67:043101

17. van Gunsteren WF, Daura X, Mark AE (2002) Helv Chim Acta 85:3113-3129

18. Hess B (2002) J Chem Phys 116:209-217

19. Baron R, Trzesniak D, de Vries AH, Elsener A, Marrink SJ, van Gunsteren WF (2007) Chem Phys Phys Chem 8:452-461

20. Krynicki K, Green CD, Sawyer DW (1978) Faraday Discuss Chem Soc 66:199-208

21. Weast RC (1986) CRC handbook of chemistry and physics. CRC Press, Boca Raton

22. Gildnyi T, Stergiopoulos C, Wolfram E (1976) Colloid Polym Sci 254:1018-1023

23. Groot RD, Rabone KL (2001) Biophys J 81:752-763

24. van der Spoel D, van Maaren PJ, Berendsen HJC (1998) J Chem Phys 108:10220-10230

25. Halle B, Wennerström H (1981) J Chem Phys 75:1928-1943

26. Cabani S, Gianni P, Mollica V, Lepori L (1981) J Solut Chem 10:563-595

27. Nagle JF, Tristram-Nagle S (2000) Biochim Biophys Acta 1469:159-195

28. Kabsch W, Sander C (1983) Biopolymers 22:2577-2637 